1 Universidade Regional do Noroeste do Estado do Rio Grande do Sul (Unijuí), Programa de Pós-Graduação Stricto Sensu em Atenção Integral à Saúde - ljuí (RS), Brasil. Universidade de Cruz Alta (Unicruz) - Cruz Alta (RS), Brasil.

daiagewehr@hotmail.com

2 Universidade Regional do Noroeste do Estado do Rio Grande do Sul (Unijuí), Programa de Pós-Graduação Stricto Sensu em Atenção Integral à Saúde - ljuí (RS), Brasil. Universidade de Cruz Alta (Unicruz) - Cruz Alta (RS), Brasil.

vanessa.acbandeira@yahoo. com.br

3 Universidade Regional do Noroeste do Estado do Rio Grande do Sul (Unijuí), Programa de Pós-Graduação Stricto Sensu em Atenção Integral à Saúde - ljuí (RS), Brasil. Universidade de Cruz Alta (Unicruz) - Cruz Alta (RS), Brasil.

gabriela.gelatti@hotmail. com

4 Universidade Regional do Noroeste do Estado do Rio Grande do Sul (Unijuí), Departamento de Ciências da Vida - ljuí (RS), Brasil. Universidade Federal do Rio Grande do Sul (UFRGS) - Porto Alegre (RS), Brasil. chriscolet@yahoo.com.br

5 Universidade Regional do Noroeste do Estado do Rio Grande do Sul (Unijuí), Departamento de Ciências da Vida - Ijuí (RS), Brasil. Universidade Federal do Rio Grande do Sul (UFRGS) - Porto Alegre (RS), Brasil. karla@unijui.edu.br

\section{Adesão ao tratamento farmacológico da hipertensão arterial na Atenção Primária à Saúde}

\author{
Adherence to pharmacological treatment of arterial hypertension in \\ Primary Health Care
}

Daiana Meggiolaro Gewehr1, Vanessa Adelina Casali Bandeira², Gabriela Tassotti Gelatti Christiane de Fátima Colet ${ }^{\mathbf{4}}$, Karla Renata de Oliveira ${ }^{5}$

RESUMO Objetivou-se verificar a adesão ao tratamento farmacológico da hipertensão arterial e fatores associados à baixa adesão em hipertensos adstritos à Atenção Primária à Saúde. Estudo transversal, realizado em duas Estratégias Saúde da Família em um município da região Noroeste do estado do Rio Grande do Sul. A coleta de dados foi realizada em domicílio no período de janeiro a maio de 2016. Para verificar a adesão ao tratamento, utilizou-se o Brief Medication Questionnaire. Participaram do estudo 145 hipertensos de ambos os sexos. Identificaram-se valores pressóricos mais elevados entre os hipertensos com baixa adesão do que entre os aderentes. Em relação à associação de medicamentos anti-hipertensivos, verificou-se que quanto maior o número de medicamentos utilizados, menor a adesão. Os fatores relacionados com a diminuição da adesão foram: baixa renda, uso de dois ou mais anti-hipertensivos e dificuldades para ler a embalagem dos medicamentos.

PALAVRAS-CHAVE Adesão à medicação. Anti-hipertensivos. Atenção Primária à Saúde. Doença crônica.

ABSTRACT The objective was to verify adherence to the pharmacological treatment of arterial hypertension and factors associated with the low adhesion of hypertensive people attached to Primary Health Care. Cross-sectional study, carried out in two Family Health Strategies in a municipality in the northwest region of RS. Data collection was carried out at home during the period of January-May 2016. The Brief Medication Questionnaire was used to check adherence to treatment. The study included 145 hypertensive of both sexes. Higher blood pressure values were identified among hypertensive patients with lower adherence than among adherents. Regarding the association of antihypertensive drugs, it was found that the higher the number of medications used, the lower the adherence. The factors related to decreased adherence were: low income, use of two or more antihypertensive drugs and difficulty reading the packaging of medications.

KEYWORDS Medication adherence. Antihypertensive agents. Primary Health Care. Chronic disease. 


\section{Introdução}

O cuidado dos usuários com doenças crônicas é um dos desafios das equipes de Atenção Primária à Saúde (APS), visto que são condições multifatoriais, com determinantes biológicos e socioculturais e com aumento proporcional do envelhecimento ${ }^{1}$. Entre essas doenças, a Hipertensão Arterial Sistêmica (HAS) é a mais prevalente. Uma revisão sistemática com estudos de 90 países demonstrou prevalência mundial de 31,3\% na população adulta ${ }^{2}$. No Brasil, de acordo com dados da Vigilância de Fatores de Risco e Proteção para Doenças Crônicas por Inquérito Telefônico ${ }^{3}$, a prevalência de HAS é de $24,8 \%$, com variação conforme a faixa etária estudada entre $22,0 \%$ na população acima de 18 anos e $69,9 \%$ nos idosos ${ }^{4,5}$.

O tratamento medicamentoso geralmente é iniciado com um ou dois anti-hipertensivos, e gradativamente podem ser associados outros medicamentos, o que pode contribuir para diminuir a adesão ao tratamento, como verificado em estudo realizado com hipertensos cadastrados em uma Unidade Básica de Saúde (UBS) em Porto Alegre, Rio Grande do Sul6.

O controle da Pressão Arterial (PA), além de exigir a participação individual, também requer a assistência da equipe de saúde, dentro de um programa eficiente de controle da HAS, pois há fatores como a cronicidade da doença, aliada à falta de sintomatologia, que influenciam e condicionam o processo do efetivo controle dos níveis pressóricos ${ }^{7}$.

A adesão ao tratamento de uma doença consiste em seguir o que foi proposto pelos profissionais de saúde. No que se refere ao tratamento farmacológico, a não adesão significa o abandono do uso dos medicamentos, sem orientação médica ou a execução de forma irregular do tratamento, seja na prática de atrasar a tomada do medicamento ou de realizar pequenas interrupções da terapêutica prescrita. A baixa adesão ao tratamento é um dos principais fatores para a persistência de valores elevados da $\mathrm{PA}^{\mathbf{8}}$.
Os profissionais de saúde, para atuarem de forma eficaz, com proposição e implementação de ações que atendam às reais necessidades dessa população, precisam conhecer os usuários e identificar os fatores da falta de adesão ao tratamento ${ }^{9}$. Nesse sentido, a detecção da conduta não aderente é fundamental para a investigação do seu impacto nos desfechos clínicos.

Diante do exposto, objetivou-se verificar a adesão ao tratamento farmacológico da HAS e fatores associados à baixa adesão em hipertensos adstritos à APS.

\section{Métodos}

Trata-se de um estudo com delineamento transversal exploratório, realizado em duas Estratégias Saúde da Família (ESF) em um município da região Noroeste do estado do Rio Grande do Sul. Este estudo respeitou os preceitos éticos para pesquisas envolvendo seres humanos e foi aprovado pelo Comitê de Ética em Pesquisa sob o Parecer Consubstanciado ${ }^{\circ}$ 1.381.719/2015.

Segundo dados fornecidos pela Secretaria Municipal de Saúde do município em estudo (2015), eram adstritos às duas ESF 5.193 usuários. Para cálculo da amostra, utilizou-se a prevalência de HAS de $24,80 \%$ segundo o Vigitel ${ }^{3}$, com nível de confiança de $95 \%$ e erro amostral de 5\%. Chegou-se ao valor de 156 hipertensos de ambos os sexos, com idade de 55 anos ou mais.

A seleção das ESF foi de modo intencional, já a seleção dos participantes da pesquisa foi realizada de modo aleatório, pelo acesso aos prontuários dos usuários adstritos às duas ESF, desse modo, considerou a prescrição de, no mínimo, um anti-hipertensivo para incluir o usuário na pesquisa. Foram excluídos os hipertensos que não tinham condições cognitivas para verbalizar e entender o questionário. Os questionários foram aplicados por uma única pesquisadora, no período de janeiro a maio de 2016. Foram realizadas três 
tentativas em horários alternados, aqueles que não foram encontrados ou não aceitaram participar da pesquisa foram excluídos do estudo.

Após a assinatura do Termo de Consentimento Livre e Esclarecido, foi aplicado, no domicílio, um instrumento de coleta de dados, com questões referentes a dados sociodemográficos e uso de medicamentos. Para verificar a adesão ao tratamento, foi utilizado o teste Brief Medication Questionnaire (BMQ) na versão em português ${ }^{6}$.

A partir do BMQ, os hipertensos foram classificados quanto à adesão ao tratamento: os hipertensos que não apresentaram respostas positivas às perguntas no teste foram classificados como aderentes; caso houvesse uma resposta positiva em um dos três domínios, foram classificados como provável aderente; se apresentaram respostas positivas em dois dos três domínios, foram classificados como provável baixa adesão; e os que apresentaram resposta positiva nos três domínios foram classificados como baixa adesão.

Para fins de análise, os resultados do BMQ foram dicotomizados, e os hipertensos foram classificados em dois grupos: os que tiveram de zero a um ponto nos três domínios foram classificados como alta adesão, e os que apresentaram pontuação de dois pontos ou mais como baixa adesão.

A aferição da pressão arterial foi realizada em domicílio, por meio de esfigmomanômetro aneroide com estetoscópio devidamente calibrados. O preparo dos participantes e os procedimentos necessários foram seguidos conforme a orientações da VII Diretriz Brasileira de Hipertensão ${ }^{10}$. A partir dos valores obtidos, os participantes foram divididos em dois grupos: os que apresentaram níveis pressóricos $\leq 140 / 90 \mathrm{mmHg}$ foram classificados com PA normal, e acima desses valores como pressão não controlada.

Os dados obtidos foram compilados em tabelas por meio do Software Statistical Package for the Social Sciences (SPSS) (versão 20.0). As variáveis foram testadas quanto à sua normalidade pelo teste Kolmogorov-Smirnov. Foi realizada análise descritiva com média, frequência absoluta e desvio padrão. A análise bivariada foi realizada para exploração inicial dos dados e foi realizada por meio do Teste qui-quadrado de Pearson e utilizou-se do teste Mann-Whitney para associação das variáveis quantitativas. Calculou-se a razão de prevalência (RP) com IC95\%. Considerou-se $\mathrm{p}<0,05$ como nível de significância estatística.

A análise multivariada foi utilizada para determinar a contribuição de cada covariável para a não adesão ao tratamento da HAS. Para tanto, empregou-se a regressão logística binária, por meio do método de Wald.

\section{Resultados}

Dos 156 hipertensos selecionados a participar do estudo, 8 (5,13\%) não foram localizados em suas residências, e $2(1,28 \%)$ não aceitaram participar da pesquisa, desse modo, 145 (92,95\%) hipertensos compuseram a amostra. A idade média foi de $66,89 \pm 8,27$ anos, a média de idade entre as mulheres foi de 66,37 $\pm 9,56$ anos, e entre os homens foi de $68,04 \pm 7,98$. Observou-se que mais da metade era do sexo feminino (69,0\%), com companheiro (75\%), brancos $(85,3 \%)$, nível educacional de ensino fundamental $(74,6 \%)$ e renda familiar acima de 2 salários mínimo $(67,2 \%)$.

No que se refere à classificação da adesão ao tratamento da HAS, 96 (66,2\%) hipertensos apresentaram alta adesão, dos quais 29 (20\%) são aderentes ao tratamento, e $67(46,2 \%)$ são prováveis aderentes. Entre os hipertensos classificados como baixa adesão (49-33,8\%), 44 (30,3\%) foram classificados como provável baixa adesão, e 5 $(3,4 \%)$ como baixa adesão ao tratamento anti-hipertensivo.

Por meio da análise bivariada, verificou-se que o estado o civil e a renda familiar foram associados com a adesão ao tratamento 
da HAS, com diminuição da adesão entre aqueles que declararam não ter companheiro (a) e renda familiar mais baixa. Os hipertensos com renda familiar mais baixa apresentaram 4,17 vezes mais chances de ter baixa adesão, e os solteiros apresentam 2,66 mais chances de não aderirem ao tratamento. As demais variáveis não apresentaram relação estatística com a adesão ao tratamento, no entanto, os hipertensos que apresentaram idade mais avançada, sexo masculino, raça/ cor não branca, menores escolaridades apresentaram diminuição da adesão ao tratamento (tabela 1).

Tabela 1. Associação entre as variáveis sociodemográficas e a adesão ao tratamento anti-hipertensivo em usuários da Atenção Primária à Saúde. RS. 2016. (n=156)

\begin{tabular}{|c|c|c|c|c|c|c|}
\hline \multirow[t]{2}{*}{ Variáveis } & \multirow[b]{2}{*}{$\begin{array}{r}\text { Baixa adesão } \\
\text { n (\%) }\end{array}$} & \multirow[b]{2}{*}{$\begin{array}{r}\text { Alta adesão } \\
\mathrm{n}(\%)\end{array}$} & \multirow{2}{*}{$\begin{array}{c}\text { Análise bivariada1 } \\
\text { RP (IC 95\%) }\end{array}$} & \multicolumn{3}{|c|}{ 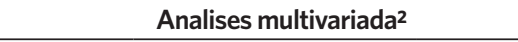 } \\
\hline & & & & $p$-value & RP (IC 95\%) & $p$-value \\
\hline \multicolumn{7}{|l|}{ Idade } \\
\hline$\geq 65$ & $48(60,0)$ & $32(40,0)$ & \multirow{2}{*}{$1,88(0,92-3,83)$} & \multirow{2}{*}{0,080} & \multirow{2}{*}{$2,20(0,93-5,17)$} & \multirow{2}{*}{0,071} \\
\hline $55-64$ & $17(26,2)$ & $48(73,8)$ & & & & \\
\hline \multicolumn{7}{|l|}{ Sexo } \\
\hline Masculino & $16(35,6)$ & $29(64,4)$ & \multirow{2}{*}{$1,12(0,53-2,35)$} & \multirow{2}{*}{0,760} & \multirow{2}{*}{$1,69(0,71-4,03)$} & \multirow{2}{*}{0,232} \\
\hline Feminino & $33(33,0)$ & $67(67,0)$ & & & & \\
\hline \multicolumn{7}{|l|}{ Cor da pele } \\
\hline Branco & $42(33,9)$ & $82(66,1)$ & \multirow{2}{*}{$1,02(0,38-2,73)$} & \multirow{2}{*}{0,962} & \multirow{2}{*}{$1,32(0,45-3,89)$} & \multirow{2}{*}{0,609} \\
\hline Não branco & $7(33,3)$ & $14(66,7)$ & & & & \\
\hline \multicolumn{7}{|l|}{ Estado civil } \\
\hline Sem companheiro & $28(46,7)$ & $32(53,3)$ & \multirow{2}{*}{$2,66(1,31-5,40)$} & \multirow{2}{*}{$0,006^{*}$} & \multirow{2}{*}{$2,11(0,927-4,804)$} & \multirow{2}{*}{0,075} \\
\hline Com companheiro & $21(24,7)$ & $64(75,2)$ & & & & \\
\hline \multicolumn{7}{|l|}{ Escolaridade } \\
\hline $\begin{array}{l}\text { NA / Ensino } \\
\text { fundamental }\end{array}$ & $45(34,4)$ & $86(65,6)$ & \multirow{2}{*}{$1,31(0,38-4,41)$} & \multirow{2}{*}{0,664} & \multirow{2}{*}{$2,419(0,57-10,25)$} & \multirow{2}{*}{0,231} \\
\hline $\begin{array}{l}\text { Ensino médio / } \\
\text { superior }\end{array}$ & $4(40,9)$ & $10(59,1)$ & & & & \\
\hline \multicolumn{7}{|l|}{ Renda Familiar } \\
\hline$\leq 2 \mathrm{SM}$ & $35(49,3)$ & $36(50,7)$ & \multirow{2}{*}{$4,17(1,98-8,77)$} & \multirow{2}{*}{$0,000^{*}$} & \multirow{2}{*}{$4,777(2,067-11,039)$} & • \\
\hline$>2 \mathrm{SM}$ & $14(18,9)$ & $60(81,1)$ & & & & 0,000 \\
\hline 1 Teste de Qui-quadrad & & & & & & \\
\hline 2 Regressão logística. & & & & & & \\
\hline NA: Não Alfabetizado; & U: Salário Mínimo & Razão de Preva & ência. & & & \\
\hline
\end{tabular}

Em relação à idade, observou-se diminuição da adesão ao tratamento entre os hipertensos com idade superior a 64 anos, a média de idade dos que foram classificados como baixa adesão foi de $67,96( \pm 8,16)$, e entre os aderentes a média foi $66,34 \pm 8,30(\mathrm{p}=0,80)$.
Os resultados da regressão logística indicaram que, entre as variáveis sociodemográficas e econômicas, a renda de um a dois salários mínimos é impactante na não adesão ao tratamento. Indivíduos com menor renda apresentaram razão de prevalência de 4,777 
A dificuldade de ler a embalagem dos medicamentos foi um dos fatores que impactaram para a não adesão ao tratamento. Dos hipertensos classificados com baixa adesão, $32,7 \%$ (16) relataram apresentar dificuldade de identificar o nome dos medicamentos e outras informações na embalagem, enquanto $14 \%$ (14) dos hipertensos aderentes apresentaram essa dificuldade $(\mathrm{p}<0,00)$ (tabela 2$)$.

Quando considerado o número de fármacos para HAS, observou-se que o grupo com níveis pressóricos superiores ao desejado utilizavam associação de mais de dois medicamentos, com média de 2,31 $\pm 1,31$, passando para $2,05 \pm 0,90$ entre os com níveis pressóricos desejado $(\mathrm{p}=0,006)$ (dados não apresentados em tabela).

Ao analisar os valores pressóricos, observou-se que 84 (57,9\%) hipertensos apresentaram níveis considerado ideal, e $61(42,1 \%)$ acima do recomendado. Entre aqueles que foram classificados como baixa adesão, 39 (79,6\%) apresentaram níveis pressóricos superiores ao recomendado. A adesão ao tratamento esteve associada ao controle pressórico com diferença estatística entre os grupos ( $\mathrm{p}=0,000)$; ademais, os não aderentes ao tratamento apresentaram risco de não controlar a PA de 13 (IC: 5,61- 30,45) vezes, superior aos aderentes (tabela 3 ).

Tabela 3. Associação entre PA, número de fármacos anti-hipertensivos em uso e a classificação e domínios do Brief Medication Questionare (BMQ). RS. 2016. $(n=156)$

\begin{tabular}{|c|c|c|c|c|}
\hline Variáveis & $\begin{array}{r}\text { PA não controlada } \\
\mathrm{n}(\%)\end{array}$ & $\begin{array}{r}\text { PA normal } \\
\mathrm{n}(\%) \\
\end{array}$ & RP (IC 95\%) & $p$-value \\
\hline Número de fármacos para HAS & & & & 0,051 \\
\hline Dois ou mais fármacos & $27(44,3)$ & $24(28,6)$ & $1,98(0,99-3,97)$ & \\
\hline Um fármaco & $34(55,7)$ & $60(71,4)$ & & \\
\hline \multicolumn{5}{|l|}{ Classificação adesão } \\
\hline Baixa adesão & $39(63,9)$ & $10(11,9)$ & $13,12(5,65-30,45)$ & $0,000^{*}$ \\
\hline Alta adesão & $22(36,1)$ & $74(88,1)$ & & \\
\hline \multicolumn{5}{|l|}{ Categorias de adesão BMQ } \\
\hline Aderente & $5(8,2)$ & $24(28,6)$ & -- & $0,000^{*}$ \\
\hline Provável aderente & $17(27,9)$ & $50(59,5)$ & & \\
\hline Provável baixa adesão & $35(57,4)$ & $9(10,7)$ & & \\
\hline Baixa adesão & $4(6,6)$ & $1(1,2)$ & & \\
\hline \multicolumn{5}{|l|}{ Domínio Regime } \\
\hline$\geq 1$ pontos & $41(69,5)$ & $18(21,4)$ & $7,51(3,56-15,85)$ & $0,000^{*}$ \\
\hline 0 ponto & $20(32,8)$ & $66(78,6)$ & & \\
\hline \multicolumn{5}{|l|}{ Domínio Crenças } \\
\hline$\geq 1$ pontos & $8(13,1)$ & $2(2,4)$ & $6,19(1,26-30,27)$ & $0,012^{\star}$ \\
\hline 0 ponto & $53(86,9)$ & $82(97,6)$ & & \\
\hline \multicolumn{5}{|l|}{ Domínio Recordação } \\
\hline$\geq 1$ pontos & $54(88,5)$ & $49(58,3)$ & $5,51(2,24-13,54)$ & $0,000^{*}$ \\
\hline O ponto & $7(11,5)$ & $35(41,7)$ & & \\
\hline
\end{tabular}

*Teste de Qui-quadrado de Pearson. HAS: Hipertensão Arterial Sistêmica. PA: Pressão Arterial; BMQ: Brief Medication Questionare. 
Entre os hipertensos com alta adesão ao tratamento, a maioria apresentou PA dentro do ideal ( $\mathrm{n}=74,88,1 \%)$, com diferença significativa entre os grupos $(\mathrm{p}=0,00)$ (tabela 3$)$. Em relação a médias da PA, o grupo com baixa adesão apresentou valores pressóricos mais elevados. A média da Pressão Arterial Sistólica (PAS) dos hipertensos com baixa e alta adesão foi respectivamente $151,43( \pm 22,3)$ $\mathrm{mmHg}, 128,0( \pm 11,64) \mathrm{mmHg}(\mathrm{p}=0,00)$. Em relação à pressão arterial diastólica (PAD), o grupo com baixa adesão apresentou média de $89,4( \pm 14,4)$, e entre os hipertensos aderentes a média foi de 78,6 $( \pm 8,0)(p=0,00)$.

Em relação aos domínios que compõem o BMQ, verificou-se que ocorreu relação entre os três domínios com o controle ou não da PA, indicando que a obtenção de escore $\geq 1$ ponto pode estar influenciando para o não controle da PA. O domínio recordação obteve maior número de respostas positivas (escore $\geq 1$ ponto) ( $\mathrm{n}=103$ - 71\%), seguido do domínio regime ( $\mathrm{n}=59$ $40,7 \%$ ) e domínio crenças ( $\mathrm{n}=10-6,9 \%$ ).

Entre as questões abordadas no BMQ no domínio recordação, 90 (62,07\%) hipertensos recebem um esquema posológico de duas ou mais vezes ao dia; desses, 47 (85,5\%) estão com os níveis pressóricos elevados; ainda, 16 (11,03\%) hipertensos relataram dificuldade em lembrar de tomar os medicamentos; desses, 12 (19,7\%) apresentam valores pressóricos acima do recomendado.

Em relação ao domínio regime, 40 (27,58\%) hipertensos destacaram a dificuldade em listar os medicamentos da HAS em uso, desses, 29 (47,5\%) apresentaram PA não controlada. 24 (16,55\%), relataram omissão de doses de algum medicamento, desses, $18(29,5)$ apresentaram níveis pressóricos superiores ao recomendado, ainda, 8 $(13,1 \%)$ relataram ter interrompido a terapia; desses, todos estavam com níveis pressóricos elevados. No domínio crenças, 6 (4,14\%) hipertensos relataram que o medicamento utilizado para hipertensão não funciona bem, e $3(4,9 \%)$ relataram algum incomodo relacionado com a medicação.

\section{Discussão}

A adesão ao tratamento farmacológico envolve diferentes elementos que constituem esse processo: o indivíduo, o tratamento, a doença, os serviços, os profissionais de saúde, bem como o meio social e cultural do usuário e de sua família. Para que a adesão seja alcançada, são necessários o alinhamento e a organização desses elementos ${ }^{\mathbf{9}, 11}$. Neste estudo, mais da metade dos hipertensos foi classificada como aderentes ao tratamento, esses apresentaram melhores níveis pressóricos, quando comparado aos com baixa adesão. Em estudo realizado por Barreto, Matsuda e Marcon'12 com 392 hipertensos adstritos à uma UBS de um município do Sul do Brasil, identificou-se que $44,90 \%$ não adeririam ao tratamento, e destes, $88,02 \%$ não apresentavam valores pressóricos em níveis desejáveis, além disso, os indivíduos não aderentes ao tratamento tiveram 9 (IC95\% 6,74 - 12,07) vezes mais chances de apresentar descontrole da PA.

Ainda, estudo internacional também demostra que a PA é de difícil controle. Estudo realizado em Coimbra, Portugal, em unidades de saúde familiar, identificou que dos 972 participantes, $35,3 \%$ não obtiveram controle da HAS ${ }^{\mathbf{1 3}}$. Nesse sentido, infere-se que a adesão ao tratamento é um dos fatores fundamentais para o controle pressórico, o que evidencia a sua importância no tratamento para o controle da PA e redução do risco cardiovascular e cerebrovascular ${ }^{14}$.

O BMQ, por estar estruturado em domínios, permite identificar as barreiras que diminuem a adesão ao tratamento. Neste estudo, questões relacionadas com as múltiplas tomadas de medicamentos, falha de dias e doses, e ter dificuldades em lembrar de tomar os medicamentos foram as mais frequentes. Além disso, os três domínios foram associados ao controle pressórico, desse modo, pode-se, por meio da aplicação desse instrumento, planejar ações. Assim, essa avaliação pode indicar quais usuários devem 
receber reforços educativos, assistência farmacêutica e atendimento multidisciplinar e quais precisam de adequação nos esquemas terapêuticos ${ }^{6}$.

Um estudo realizado em Curitiba, Paraná, para avaliar a adesão ao tratamento farmacológico em adultos hipertensos, utilizando o mesmo instrumento deste estudo, identificou $59 \%$ da amostra com escore compatível com provável baixa adesão. A maioria das repostas positivas foi nos domínios recordação (61\%), do domínio regime (55\%) e crenças $(9,33 \%)$ respectivamente ${ }^{15}$. Do mesmo modo, em estudo realizado por Vancini-Campanharo et al. ${ }^{16}$ com hipertensos no Serviço de Emergência do Hospital São Paulo, constatou-se que $56 \%$ dos usuários apresentaram adesão moderada ao tratamento, sendo que a principal barreira a essa adesão foi a recordação em relação ao uso do medicamento, encontrada em $67 \%$.

Destaca-se que as características socioeconômicas exercem grande influência sobre a adesão ao tratamento da HAS. A baixa escolaridade e a renda podem interferir negativamente nesse processo; essas características são frequentes principalmente entre os idosos, o que ficou evidente na amostra estudada. Nesse sentido, Barreto et al.12 verificaram que $63,51 \%$ dos entrevistados possuíam baixos níveis educacionais, e a não adesão à farmacoterapia foi significativamente mais frequente entre estes, ainda, indivíduos com HAS que possuíam 8 anos ou menos de estudo apresentaram chance 1,3 vez maior de não aderirem à farmacoterapia, dados que corroboram o presente estudo (tabela 1).

Em relação ao estado civil, os hipertensos com companheiro aderiram mais ao tratamento, com ocorrência de diferença entre os grupos. Em estudo realizado com idosos em um ambulatório de especialidades médicas em Vitória, Espírito Santo, verificou que ocorreu maior adesão ao tratamento entre os idosos que residiam com companheiro ${ }^{17}$.

Constata-se neste estudo que, com o avançar da idade, a adesão ao tratamento diminui, assim como observado por Barreto, Matsuda e Marcon ${ }^{12}$ em trabalho realizado com indivíduos hipertensos em tratamento ambulatorial na APS de um município da região Sul. Além disso, os idosos tendem a apresentar diversas vulnerabilidades, como declínio das capacidades físicas, mentais e cognitivas, o que dificulta a compreensão e a adesão ao tratamento farmacológico e não farmacológico prescritos ${ }^{18}$.

No presente estudo, o tempo de tratamento não influenciou na adesão e não se revelou com risco aos usuários, mas foi observado que hipertensos com maior tempo de diagnóstico e tratamento foram menos aderentes, o que corrobora a VII Diretriz Brasileira de Hipertensão ${ }^{10}$. Esses autores inferem que a diminuição da adesão ao tratamento da HAS aumenta com o tempo de tratamento, devido principalmente ao seu caráter crônico. Ainda, Mantovani et al. ${ }^{15}$ verificaram que um maior tempo de diagnóstico implica menor adesão. Nesse sentido, reforça-se a necessidade de acompanhamento dos hipertensos, independentemente do tempo de diagnóstico da HAS, uma vez que não há consenso em relação aos estudos analisados.

O uso de associação de fármacos para o controle da HAS mostrou-se relacionado com maiores níveis pressóricos, visto que o aumento do número de medicamentos contribuiu para diminuição da adesão, além disso, comorbidades e não adesão às medidas não farmacológicas podem contribuir para associação de medicamentos e não ser eficaz para o controle da PA. O estudo realizado por Barreto, Matsuda e Marcon'12 revelou que indivíduos com prescrições medicamentosas de três ou mais fármacos tiveram 1,2 (IC: 1,13 - 1,35) vez maior chance de apresentar baixo controle pressórico, quando comparados com aqueles que faziam uso de até dois medicamentos. Ainda, em um estudo que avaliou a adesão pelo método do $B M Q$, identificou-se alta correlação entre o número de fármacos utilizados e a barreira 
de recordação, desse modo, quando é preciso associações de medicamentos, é necessário que a equipe busque estratégias juntamente com o paciente para diminuir a taxa de esquecimento ${ }^{16}$. Essas constatações demonstram a importância da realização de acompanhamento farmacoterapêutico no paciente hipertenso, especialmente naqueles que fazem uso de vários fármacos associados.

Combinações podem ser realizadas com fármacos em separado ou em associação fixa. Se por um lado o uso de combinações livres permite a escolha da dose de cada um dos componentes, por outro, verificou-se que o uso de associações fixas favorece a adesão à terapêutica, pelo uso de menor número de comprimidos ${ }^{10}$. Os achados apontam a necessidade de estratégias para garantir o acesso a tratamentos que possibilitem diminuir o número de medicamentos prescritos e o número de administrações diárias, uma estratégia importante seria a oferta pelo Sistema Único de Saúde (SUS) 1 .

Os resultados evidenciaram que o acesso aos medicamentos não está relacionado com a baixa adesão ao tratamento, desse modo, levam a crer que o SUS vem alcançando seus objetivos no que se refere ao acesso dos usuários aos fármacos ${ }^{19}$. O acesso gratuito a medicamentos tem sido garantido por vários programas, apesar disso, surge uma lacuna quando se avalia a adesão: muitos hipertensos, mesmo com acesso aos medicamentos, não sabem como utilizá-los corretamente, o que coloca em risco a efetividade do tratamento prescrito e contraria as políticas de uso racional de medicamentos ${ }^{20}$.

Nesse sentido, recomenda-se, preferencialmente, que os usuários sejam acompanhados por equipe multidisciplinar e que seus familiares sejam envolvidos em todo o processo, o que aumenta as taxas de adesão e as chances de sucesso com o tratamento ${ }^{10}$. Um estudo realizado com hipertensos evidenciou que maiores conhecimentos em relação à gravidade da HAS podem resultar em maior adesão no domínio regime ${ }^{16}$. Desse modo, o conhecimento sobre a doença se constitui em uma intervenção para melhoria da adesão, com ações educativas centradas nas crenças e inquietações sobre suas condições de saúde e de tratamento.

Ainda, recomenda-se que as ações educativas sejam desenvolvidas de modo multidisciplinar, com atuação de forma integrada na abordagem da avaliação de risco, adoção de medidas de promoção da saúde e atendimento aos usuários dos serviços de acompanhamento ambulatorial17. Nesse sentido, a elaboração de protocolos terapêuticos, com a padronização de ações que resultem em melhores cuidados e adesão ao tratamento é essencial.

Do mesmo modo que o acompanhamento farmacoterapêutico é fundamental para a gestão compartilhada do tratamento entre profissionais e usuários e possibilita a adoção de estratégias voltadas para necessidades individuais específicas e que envolvam o usuário como participante no manejo de sua saúde ${ }^{1,21}$. Uma metanálise mostrou que as intervenções farmacêuticas podem melhorar significativamente a aderência à medicação, PAS, PAD e controle da PA em usuários com hipertensão ${ }^{21}$. Em ensaio clínico randomizado desenvolvido durante nove meses com hipertensos em Portugal, a intervenção farmacêutica resultou na redução significativa da PA sistólica e PA diastólica e aumento da proporção de hipertensos com PA controlada ${ }^{22}$.

Nessa perspectiva, a ESF configura-se como elemento-chave para a organização e implantação de ações que visem ao controle da HAS. Destacam-se a necessidade do trabalho multiprofissional e interdisciplinar para a abordagem ao hipertenso e o cuidado domiciliar como uma estratégia para maior adesão ao tratamento e controle da doença, para, assim, minimizar os seus impactos e os da não adesão tanto para o sistema de saúde quanto para os usuários ${ }^{23}$. Para a compreensão das barreiras da não adesão ao tratamento da HAS, é necessário entender que a 
maioria dos fatores vai além do individual, que envolve vários aspectos e que a adesão ao tratamento poderia ser mais efetiva se estratégias conjuntas fossem discutidas e implementadas por toda a equipe de saúde envolvida na terapêutica ${ }^{24 .}$

A partir da verificação da adesão ao tratamento da HAS e das barreiras envolvidas na adesão, é possível planejar ações que auxiliem a equipe de saúde a promover atividades direcionadas aos usuários não aderentes e que também reforcem as orientações aos aderentes. Nesse sentido, além do acompanhamento farmacoterapêutico, é necessário implementação de medidas que aumentem a adesão às medidas não farmacológicas. Desse modo, mudanças no estilo de vida, embora difíceis de alcançar, não só podem retardar a taxa de desenvolvimento da hipertensão, mas também apresentam benefício adicional em reduzir outros fatores de risco cardiovascular, no entanto, tais medidas preventivas continuam recebendo prioridade relativamente baixa, em que a ênfase principal ainda está sendo colocada no tratamento farmacológico da doença estabelecida ${ }^{25}$.

Como limitações deste estudo, destaca-se que a aferição da PA foi realizada em um único momento, o que pode não representar a real condição do usuário. Além disso, o método de adesão empregado baseia-se em respostas autorreferidas, desse modo, os resultados podem sofrer interferência de vieses como o de recordação e de desejabilidade social, especialmente quando se trata de questões relacionadas com o tratamento farmacológico da HAS.

\section{Conclusões}

A baixa adesão ao tratamento farmacológico da HAS foi constatada em menos da metade dos indivíduos acompanhados pela APS, o que irá comprometer o sucesso terapêutico, pois a não adesão está entre os fatores que afetam o controle pressórico, e, desse modo, tende a aumentar as complicações da HAS não controlada.

Os fatores relacionados com a diminuição da adesão ao tratamento foram: baixa renda, uso de dois ou mais anti-hipertensivos e ter dificuldades em ler a embalagem dos medicamentos. Não foi verificada associação entre a adesão ao tratamento e o acesso aos medicamentos, o que permite inferir que o acesso a eles não é um problema para o usuário, por outro lado, o uso correto dessa e de outras estratégias terapêuticas para o controle da HAS se fazem necessárias, o que evidencia a importância da organização e planejamento da equipe para a organização do cuidado a esses usuários, incluindo o acompanhamento farmacoterapêutico. 


\section{Referências}

1. Tavares NUL, Bertoldi AD, Thume E et al. Fatores associados a baixa adesão ao tratamento medicamentoso em idosos. Rev Saúde Pública [internet]. 2013 [acesso em 2016 jan 2]; 47(6):1092-101. Disponível em: http://www.scielo.br/scielo.php?script=sci arttext\&pid=S0034-89102013000601092\&lng=en\& nrm=iso\&tlng=pt.

2. Mills KT, Bundy JD, Kelly TN, et al. Global Disparities of Hypertension Prevalence and ControlClinical Perspective. Circulation [internet]. 2016 [acesso em 2016 nov 5];134(6):441-50. Disponível em: http://circ.ahajournals.org/lookup/ doi/10.1161/CIRCULATIONAHA.115.018912.

3. Brasil. Ministério da Saúde. Secretaria de Vigilância em Saúde. Vigitel Brasil 2013: Vigilância de fatores de risco e proteção para doenças crônicas por inquérito telefônico. Brasília, DF: MS; 2014.

4. Malta DC, Santos NB, Perillo RD, et al. Prevalence of high blood pressure measured in the Brazilian population, National Health Survey, 2013. São Paulo Med J [internet]. 2016 [acesso em 2016 nov 8]; 134(2):16370. Disponível em: http://www.scielo.br/pdf/spmj/ v134n2/1806-9460-spmj-134-02-00163.pdf.

5. Pimenta FB, Pinho L, Silveira MF, et al. Fatores associados a doenças crônicas em idosos atendidos pela Estratégia de Saúde da Família. Ciênc Saúde Colet [internet]. 2015 [acesso em 2016 out 5]; 20(8):24892498. Disponível em: http://www.scielo.br/pdf/ csc/v20n8/1413-8123-csc-20-08-2489.pdf.

6. Ben AJ, Neumann CR, Mengue SS. Teste de Morisky-Green e Brief Medication Questionnaire para avaliar adesão a medicamentos. Rev Saúde Publica [internet]. 2012 [acesso em 2016 nov 5]; 46(2):279-89. Disponível em: http://www.scielo.br/ pdf/rsp/v46n2/3357.pdf.

7. Barreto MS, Cremonese IZ, Janeiro V, et al. Prevalência de não adesão à farmacoterapia anti-hipertensiva e fatores associados. Rev Bras Enferm [internet]. 2015 Fev [acesso em 2016 set 21];
68(1):60-7. Disponível em: http://www.scielo.br/ scielo.php?script=sci_arttext\&pid=S003471672015 000100060\&lng=pt\&nrm=iso\&tlng=en.

8. Mancia G, Fagard R, Narkiewicz K, et al. Guidelines de 2013 da ESH/ESC para o Tratamento da Hipertensão Arteriale. Rev Port Hipertens e Risco Cardiovasc [internet]. 2014 Jan-Fev [acesso em 2016 out 17]; 39. Disponível em: https://www.sphta. org.pt/files/guidelines_31janeiro2014-final.pdf.

9. Reiners AAO, Seabra FMF, Azevedo RCS, et al. Adesão ao tratamento de hipertensos da atenção básica. Ciênc Cuid Saúde [internet]. 2012 [acesso em 2016 set 17]; 11(3):581-587. Disponível em: http://periodicos.uem.br/ojs/index.php/CiencCuidSaude/ article/view/16511/pdf.

10. Malachias MVB, Souza WKSB, Rodrigues CIS, et al. $7^{\text {a }}$ Diretriz brasileira de hipertensão arterial. Arq Bras Cardiol. 2016 [acesso em 2016 set 17]; 107(3):1-103. Disponível em: http://publicacoes.cardiol.br/2014/diretrizes/2016/05_HIPERTENSAO_ ARTERIAL.pdf.

11. Motta PG, Carvalho GG, Faioli MA, et al. Adesão medicamentosa ao tratamento da hipertensão de pacientes do hiperdia em Ipatinga e Timóteo, Minas Gerais. Rev Uningá [internet]. 2014 [acesso em 2016 set 18]; 40(1):91-103. Disponível em: https://www.mastereditora.com.br/periodico/20140723_184536.pdf.

12. Barreto MS, Matsuda LM, Marcon SS. Fatores associados ao inadequado controle pressórico em pacientes da atenção primária. Esc Anna Nery Rev Enferm [internet]. 2016 [acesso em 2016 nov 11]; 20(1):114-20. Disponível em: http://www.gnresearch.org/doi/10.5935/1414-8145.20160016.

13. Santiago LM, Pereirab C, Botasb P, et al. Pacientes com hipertensão arterial em ambiente de medicina geral e familiar: análise comparativa entre controlados e não controlados. Rev Port cardiol [internet]. 2014 [acesso em 2016 set 12]; 33(7-8):419-424. Disponível em: https://www.sciencedirect.com/ 
science/article/pii/S0870255114000353.

14. Souza FFR, Andrade KVF, Sobrinho, CLN. Adesão ao tratamento farmacológico e controle dos níveis pressóricos de hipertensos acompanhados na Estratégia de Saúde da Família. Rev Bras Hipertens [internet]. 2015 [acesso em 2016 nov 22]; 22(4):133138. Disponível em: http://departamentos.cardiol. br/sbc-dha/profissional/revista/22-4.pdf.

15. Mantovani MF, Mattei AT, Arthur JP, et al. Utilização do brief medication questionnaire na adesão medicamentosa de hipertensos. Rev enferm UFPE [internet]. 2015 [acesso em 2016 out 10]; 9(1):84-90. Disponível em: https://periodicos.ufpe.br/revistas/ revistaenfermagem/article/view/10310/0.

16. Vancini-Campanharo CR, Oliveira GN, Fernanda T, et al. Hipertensão Arterial Sistêmica no Serviço de Emergência: adesão medicamentosa e conhecimento da doença. Rev. Latino-Am. Enfermagem [internet]. 2015 [acesso em 2016 out 12]; 23(6). Disponível em: http://www.scielo.br/pdf/rlae/v23n6/pt_01041169-rlae-23-06-01149.pdf.

17. Arruda DCJ, Eto FN, Velten APC, et al. Fatores associados a não adesão medicamentosa entre idosos de um ambulatório filantrópico do Espírito Santo. Rev Bras Geriatr e Gerontol [internet]. 2015; [acesso em 2016 out 12]; 18(2):327-37. Disponível em: http://www.scielo.br/pdf/rbgg/v18n2/1809-9823rbgg-18-02-00327.pdf.

18. Zavatini MA, Obreli-Neto PR, Cuman RKN. Estratégia saúde da família no tratamento de doenças crônico-degenerativas: avanços e desafios. Rev Gaúcha Enferm [internet]. 2010 [acesso em 2016 nov 12]; 31(4):647-54. Disponível em: http://seer.ufrgs.br/index.php/RevistaGauchadeEnfermagem/ article/view/13275/11845.

19. Aziz MM, Calvo MC, Schneider IJC, et al. Prevalência e fatores associados ao acesso a medicamentos pela população idosa em uma capital do sul do Brasil: um estudo de base populacional. Cad Saúde Pública [internet]. 2011 [acesso em 2016 out 11]; 27(10):1939-1950. Disponível em: http://www. scielo.br/pdf/csp/v27n10/07.pdf.

20. Motter FR, Teresa M, Olinto A, et al. Conhecimento sobre a farmacoterapia por portadores de Hipertensão Arterial Sistêmica. Ciênc Saúde Colet [internet]. 2013 [acesso em 2016 set 12]; 18(8):2263-2274. Disponível em: http://www.scielo.br/pdf/csc/v18n8/10.pdf.

21. Morgado M, Rolo S, Castelo-branco M. Pharmacist intervention program to enhance hypertension control: a randomised controlled trial. Int J Clin Pharm [intener]. 2011 [acesso em 2016 out 16]; 33:132-140. Disponível em: https://www.ncbi.nlm. nih.gov/pmc/articles/PMC3042105.

22. Morgado MP, Morgado SR, Mendes LC, et al. Pharmacist interventions to enhance blood pressure control and adherence to antihypertensive therapy: Review and meta-analysis. Am J Heal Pharm [intenet]. 2011 [acesso em 2016 out 12]; 68:241-254. Disponível em: http://www.ajhp.org/ content/68/3/241.long?sso-checked=true.

23. Ribeiro AG, Cotta RMM, Silva LS, et al. Hipertensão arterial e orientação domiciliar: o papel estratégico da saúde da família. Rev Nutr [internet]. 2012 [acesso em 2016 nov 15]; 25(2):271-282. Disponível em: http://www.scielo.br/scielo.php?script=sci_arttext \&pid=S1415-52732012000200009.

24. Silva AP, Avelino FVSD, Sousa CLA, et al. Fatores associados à não adesão ao tratamento da hipertensão arterial sistêmica: uma revisão integrativa. Rev Pesqui Cuid Fundam (online) [internet]. 2016 [acesso em 2016 out 12]; 8(1):4047-4055. Disponível em: http://www.seer.unirio.br/index.php/cuidado fundamental/article/view/5019/pdf_1834.

25. Chobanian AV. Guidelines for the Management of Hypertension. Med Clin [internet]. 2017 [acesso em 2016 out 21]; 101(1):219-227. Disponível em: http:// dx.doi.org/10.1016/j.mcna.2016.08.016.

Recebido em 06/07/ 2017

Aprovado em 08/02/2018

Conflito de interesses: inexistente

Suporte financeiro: não houve 